TITLE:

\title{
Mode switching of an optical motor
}

$\operatorname{AUTHOR}(\mathrm{S})$ :

Harada, T; Yoshikawa, K

CITATION:

Harada, T ...[et al]. Mode switching of an optical motor. APPLIED PHYSICS LETTERS 2002, 81(25): 4850-4852

ISSUE DATE:

2002-12-16

URL:

http://hdl.handle.net/2433/49849

\section{RIGHT:}

Copyright 2002 American Institute of Physics. This article may be downloaded for personal use only. Any other use requires prior permission of the author and the American Institute of Physics. 


\title{
Mode switching of an optical motor
}

\author{
Takahiro Harada and Kenichi Yoshikawa ${ }^{a}$ \\ Department of Physics, Graduate School of Science, Kyoto University and CREST, Kyoto, 606-8502, Japan
}

(Received 15 April 2002; accepted 11 October 2002)

\begin{abstract}
A dielectric object irradiated with a strongly focused laser beam is affected by the trapping force as well as the scattering force. When the object is chirally shaped, it can be rotated in a certain direction. Here, we show that the rotational direction is controlled as desired only by changing the position of the focal spot on the same chiral object. We show the experimental evidence of the mode switching by using a helical chromosome under steady illumination of a $\mathrm{Nd}^{3+}$ :YAG laser beam. (c) 2002 American Institute of Physics. [DOI: 10.1063/1.1527235]
\end{abstract}

It is a challenging task to construct a rotating motor of a micron scale that works at a desired speed and direction under remote control. In the past two decades, it has been well known that a focused laser beam can be used to trap and manipulate microparticles in an aqueous environment. This technique has been also applied to make microparticles rotate in a certain direction. Ashkin suggested that rotation will be induced on a micro-object optically levitated with a focused laser beam that has a circular polarization. ${ }^{1}$ However, single beam trapping is unstable in this scheme. Dholakia et $a l^{2}$ utilized the orbital angular momentum of LagguerreGaussian beams to generate rotation. Masuhara et al. ${ }^{3}$ showed that a micro-object can be rotated with scanning laser micromanipulation. Though these techniques enable an easy switching of a rotational direction, they require complicated optical systems. There is another research stream to construct an optical rotor by utilizing a chirally shaped object to generate a torque through light scattering. ${ }^{4,5}$ On account of the chiral shape of the optically trapped object, the scattering force affords a torque to the object and rotates it around the trapping center, namely the focal spot of the light. Altough the latter mechanism enables stable high-speed rotation only by a single laser beam with simple devices, it has been regarded that the direction of the rotary motion is uniquely determined by the chirality of the object and can never be reversed on the same object.

In this letter, we show that a single object can be rotated in the desired direction, clockwise or counterclockwise. It is achieved by controlling the location of the trapping center on the rotor, without any change in the optics. In the experiment, we chose a chromosome in the sperm nucleus of it Xenopus laevis, ${ }^{6}$ an amphibian popular in biological experiments, as a chirally shaped rotor, which consists of deoxyribonucleic acid (DNA) and other proteins that are packed into a left-hand sided helical shape of $20 \mu \mathrm{m}$ length and $1 \mu \mathrm{m}$ diameter. We attached a negatively charged polystyrene bead ( $2 \mu \mathrm{m}$ diameter) to a desired position on the chromosome that is positively charged. The bead serves as a trapping center when the chromosome-bead complex is optically trapped, because the bead has a high refractive index.

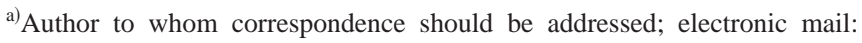
yoshikaw@scphys.kyoto-u.ac.jp
}

The chromosome lies in a preferable orientation and rotates around the light axis immediately after it has been trapped. According to the location of a bead sticking, the mode of rotation changes in a characteristic manner as shown in Fig. 1. With an attached bead as a trapping center, three different modes are observed. In mode A [Fig. 1(a)]; the chromosome rotates clockwise around the bead, which is attached to the inside end of the helix. In mode B [Fig. 1(b)]; the chromosome rotates counterclockwise around the bead, which is attached to the outside end of the helix. In mode $\mathrm{C}$ [Fig. 1(c)]; the chromosome rotates counterclockwise around the bead located near the center of the helix. The chromosome lies almost horizontally in this mode. On the contrary, in the absence of an attached bead, only a clockwise mode is generated as mode D [Fig. 1(d)], in which the chromosome is trapped by the central part. It has been confirmed that the angular velocity of the chromosome depends linearly on the power of the incident light beam in all of these modes [Fig. 2(a)].

We will subsequently discuss how the helical chromosome is trapped and rotated. The electromagnetic force on a dielectric object irradiated with a focused laser beam is approximately divided into an attracting part and a scattering part. ${ }^{7}$ In this system, while the attracting force determines the position and orientation of the chromosome, the rotation is driven by the light-scattering force due to the chiral shape of the object.

To confirm this consideration, we estimated the lightscattering torque and the drag torque in a semiquantitative manner. First, the shape of the choromosome is assumed to be a helical tube as shown in Fig. 3(a). ${ }^{8}$ In the calculation procedure, the helical tube is placed on the appropriate position to the optical cone in each mode as in the right-hand side column of Fig. 1. The intensity distribution of the focused single Gaussian beam is supposed to be $I(x, y, z)$ $=2 I_{0} / \pi\left(w_{0}^{2}+\tan ^{2} \beta z^{2}\right) \cdot \exp \left\{-2\left(x^{2}+y^{2}\right) /\left(w_{0}^{2}+\tan ^{2} \beta z^{2}\right)\right\}$, where $w_{0}$ is the radius of the minimum beam waist, $\beta$ is the half angle of the optical cone, and $I_{0}$ is the power of the incident beam. The light-scattering force is calculated as follows. At each small part of the helical tube, denoted by $\theta$, the scattering ray is determined by the laws of ray optics ${ }^{9}$ for the given incident light ray. The difference between the momenta of the scattering and the incident rays is conveyed to the tube as optical force [Fig. 3(b)]. The torque about the rotational 


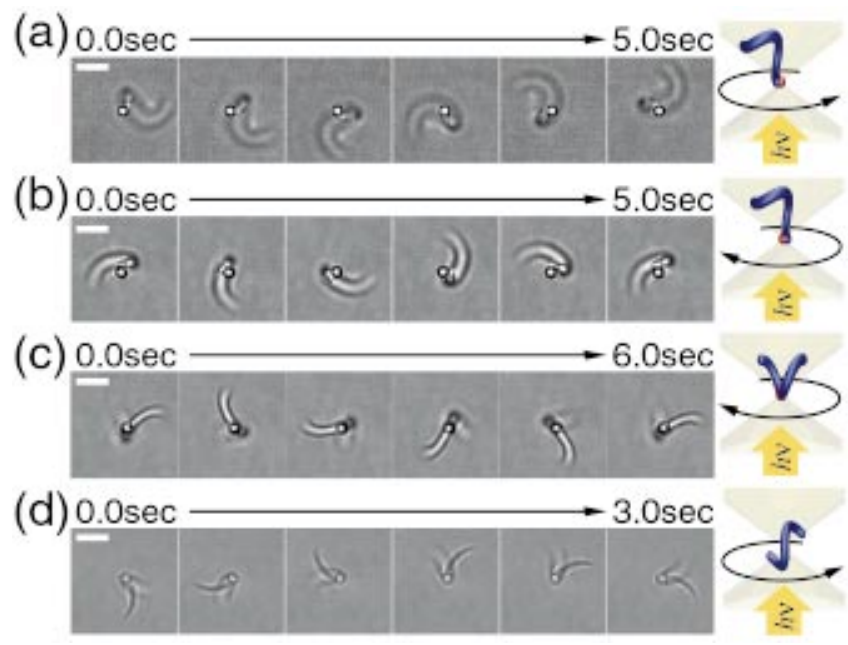

FIG. 1. (Color) Various modes of rotation of the left-hand sided helical chromosomes. The series of microscopic images of the rotating chromosomes (the bars represent $5 \mu \mathrm{m}$ ), together with the schematic images of the side view. (a) Mode A: Clockwise rotation, where an optically trapped bead locates inside the end of the helical chromosome. (b) Mode B: Counterclockwise rotation, where a trapped bead locates outside the end of the helix. (c) Mode C: Counterclockwise rotation, where the trapped bead locates near the center of the helix. (d) Mode D: Clockwise rotation, where the helix itself is optically trapped by its center. The powers of the incident beam were $10 \mathrm{~mW}$ in (a) and (b), and $20 \mathrm{~mW}$ in (c) and (d).

axis (optical axis) corresponding to this force is integrated over the whole tube to be the scattering torque $T_{S}\left(I_{0}\right)$. Similarly, the drag torque is also calculated. The drag force for the velocity at each small part of the tube is derived with the Oseen approximation ${ }^{10}$ by replacing the helical tube with a virtual long cylinder at the point. Then, the torque corresponding to that drag force is integrated over the whole tube,

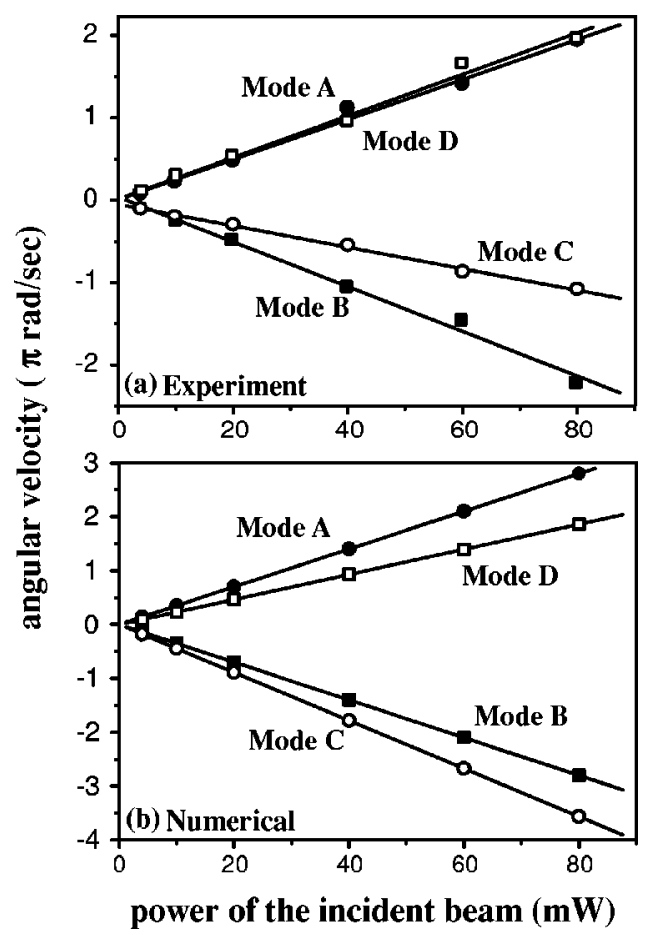

FIG. 2. Relationship between the power of the incident beam and the angular velocity of the chromosome in (a) the experiment and (b) the numerical estimation. In each graph, the closed circles, the closed squares, the open circles, and the open squares correspond to rotational modes A, B, C, and D, respectively. planes of torque.
Downloaded 06 Mar 2008 to 130.54.110.22. Redistribution subject to AIP license or

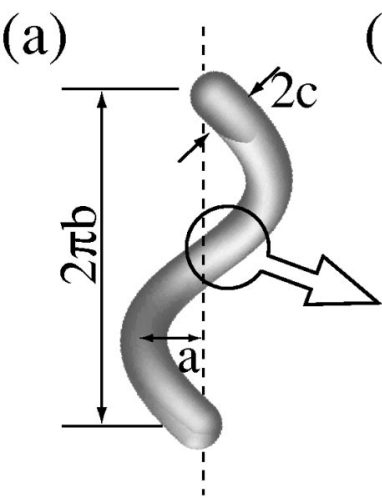

(b)

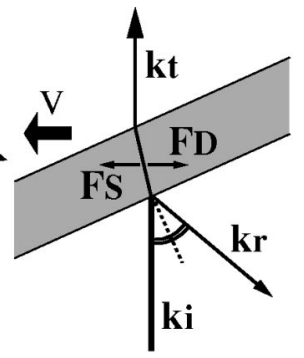

FIG. 3. Procedure on the numerical estimation of the scattering and drag torque. (a) The shape of the chromosome is assumed to be a helical tube of radius $c$ with a core given as $x=a \sin \theta, y=a \cos \theta$, and $z=b \theta(0 \leqslant \theta$ $\leqslant 2 \pi$ ). The parameters $a, b$, and $c$ are indicated in the figure. (b) For each small part of the helical tube, the scattering force is determined by ray optics. The momentum-flux vectors $\mathbf{k}_{\mathbf{i}}, \mathbf{k}_{\mathbf{t}}$, and $\mathbf{k}_{\mathbf{r}}$ denote the incident, transmitted, and reflected rays, respectively. $\mathbf{F}_{\mathbf{S}}$ is the scattering force given by $\mathbf{F}_{\mathbf{s}}=\mathbf{k}_{\mathbf{t}}+\mathbf{k}_{\mathbf{r}}-\mathbf{k}_{\mathbf{i}} . \mathbf{F}_{\mathbf{D}}$ is the drag torque from the solvent for the velocity $V$ of this small part.

and the drag torque $T_{D}(\omega)$ is obtained as a function of the angular velocity $\omega$. Since the scattering torque and the drag torque balance each other as $T_{S}\left(I_{0}\right)=T_{D}(\omega)$ at the steady rotation, we obtained the relationship between $I_{0}$ and $\omega$. The result is shown in the Fig. 2(b). The sign and the magnitude agree with the experimental trend in spite of such rough approximation.

In the aforementioned calculation, it is to be noted that the sign and magnitude of the scattering torque is almost determined by the limited region around the focus. This is because the scattering torque about the rotational axis is proportional to the local intensity of the highly focused light, the torque exerted near the focus plays a dominant role. Based
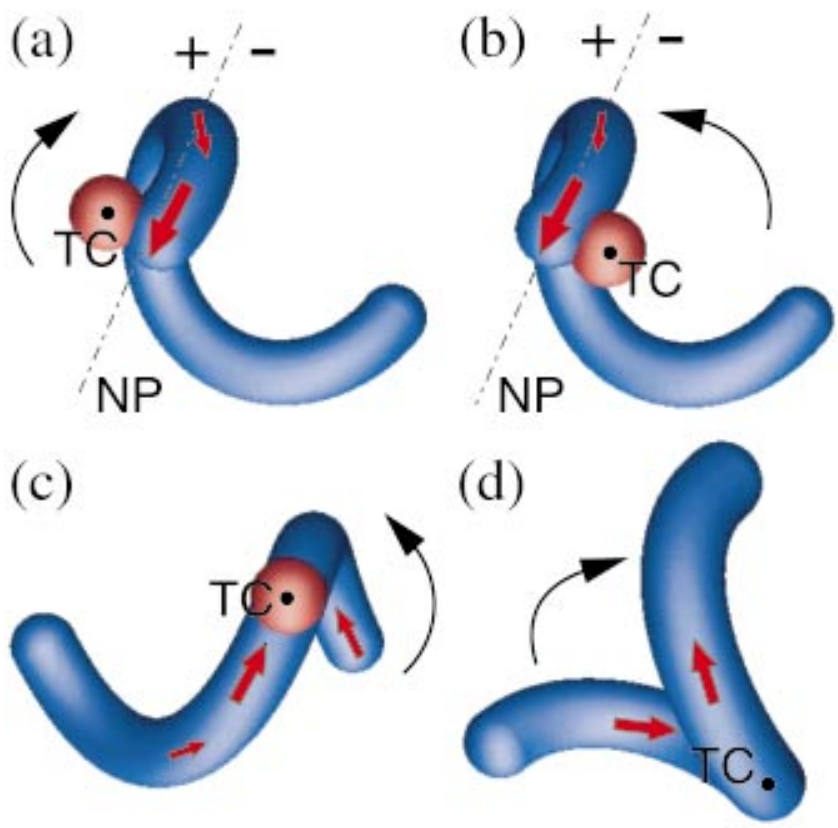

FIG. 4. (Color) The schematic images of the rotating chromosomes in (a) Mode A, (b) Mode B, (c) Mode C, and (d) Mode D. The red arrows indicate scattering force acting on the chromosomes. TCs indicate the locations of the trapping centers (focuses), and NPs represent the positions of the null 
on this consideration, the effective force acting on the chromosome is schematically shown in Fig. 4. As illustrated in Fig. 4, the scattering-force vector is along the helix of the chromosome, and the force near the focus determines the direction of rotation.

Let us discuss further why the direction of rotation is opposite between modes A and B, which have only a slight difference in the position of the attached beads. The geometry of the helical objects in these modes is close to each other, especially in the small regions within 2-3 $\mu \mathrm{m}$ from the trapping centers. Therefore, the scattering force $\mathbf{F}$, acting on these regions, denoted by $\mathbf{r}_{\mathbf{0}}$, is almost the same. Since that force $\mathbf{F}$ is nearly equal to the total force on the object as mentioned herein, the net torque in modes $\mathrm{A}$ and $\mathrm{B}$ is written as $\mathbf{T}_{\mathbf{A}} \approx\left(\mathbf{r}_{\mathbf{0}}-\mathbf{r}_{\mathbf{A}}\right) \times \mathbf{F}$ and $\mathbf{T}_{\mathbf{B}} \approx\left(\mathbf{r}_{\mathbf{0}}-\mathbf{r}_{\mathbf{B}}\right) \times \mathbf{F}$, where $\mathbf{r}_{\mathbf{A}}$ and $\mathbf{r}_{\mathbf{B}}$ are the trapping centers ("TCs" in Fig. 4) in modes A and B, respectively. These equations indicate that the signs of $\mathbf{T}_{\mathbf{A}}$ and $\mathbf{T}_{\mathbf{B}}$ are opposite because the trapping centers, $\mathbf{r}_{\mathbf{A}}$ and $\mathbf{r}_{\mathbf{B}}$, are across the force-acting point $\mathbf{r}_{\mathbf{0}}$, to put it more precisely, across a plane that is determined from $\mathbf{r}_{\mathbf{0}}, \mathbf{F}$, and the light axis. Call this plane "the null plane of torque" ("NPs" in Fig. 4).

In conclusion, it has been experimentally shown that the rotation of a single light-driven rotor can be reversed by controlling the location of the trapping center. The mode switching in the rotational motions of the rotor can be explained semiquantitatively by considering the light-scattering torque. The reversal of the rotation in the special cases (modes A and B) is also understood qualitatively in terms of the null plane of torque. Since the idea of the null plane of torque is quite general, it is promising to design and to fabricate bidirectional rotors based on this concept.

The authors would like to thank Dr. T. Akitaya and Dr. $\mathrm{K}$. Ohsumi for their generous advice on the preparation of the chromosomes.
${ }^{1}$ A. Ashkin, Science 210, 1081 (1980).

${ }^{2}$ L. Paterson, M. P. MacDonald, J. Arlt, W. Sibbett, P. E. Bryant, and K. Dholakia, Science 292, 912 (2001).

${ }^{3}$ H. Misawa, K. Sasaki, M. Koshioka, N. Kitamura, and H. Masuhara, Appl. Phys. Lett. 60, 310 (1991).

${ }^{4}$ E. Higurashi, H. Ukita, H. Tanaka, and O. Ohguchi, Appl. Phys. Lett. 64, 2209 (1994).

${ }^{5}$ P. Galajda and P. Ormos, Appl. Phys. Lett. 78, 249 (2001).

${ }^{6}$ The chromosomes were prepared by the method as described by J. B. Gurdon, J. Embryol. Exp. Morph. 36, 523 (1976). The beads attached to the chromosomes were carboxylate-modified microspheres of $2 \mu \mathrm{m}$ diameter, purchased from Molecular Probes, USA. Beads were mixed with chromosomes at the numerical ratio of 1:1 and left at room temperature for $\sim 1 \mathrm{~h}$ resulting in attachment of almost one bead to an individual chromosome. For the experiment, we picked out chromosomes associated with one bead on the desired position. The chromosomes were dispersed in $\mathrm{D}_{2} \mathrm{O}$, which has no absorption at $1064 \mathrm{~nm}$ wavelength, in order to prevent heat convection in the solvent. The apparatus consisted of a microscope (TE-300, Nikon) and a $\mathrm{Nd}^{3+}$ :YAG laser (SL902T, continuous wave in $\mathrm{TEM}_{00}$ mode at $1064 \mathrm{~nm}$ wavelength, Spectron). The laser beam was fed into the microscope after it was decreased down to various powers with a variable ND filter in order to obtain a stable beam. Nikon PlanFl $\times 100$ oil-immersion objective lens (numerical aperture 1.30 ) was used to focus the light. The motion was recorded with a charge-coupled device camera.

${ }^{7}$ K. Svoboda and S. M. Block, Annu. Rev. Biophys. Biomol. Struct. 23, 247 (1990).

${ }^{8}$ We adopted the following parameters for the calculation. The parameters for the shape of the helical tube $a, b$, and $c$ are determined as $a$ $\approx 2.5 \mu \mathrm{m}, b \approx 2.1 \mu \mathrm{m}$, and $c \approx 0.5 \mu \mathrm{m}$ by fitting microscopic images to the helix function. The radius of the attached bead is $1 \mu \mathrm{m}$. The radius of the minimus beam waist is $w_{0} \approx 0.5 \mu \mathrm{m}$. The focusing angle $\beta \approx \pi / 3$ is determined by the numerical aperture of the objective lens. As for the refractive index of the chromosome, we assumed as 1.6 following a known value for a DNA film. [See A. Wittlin, L. Genzel, F. Kremer, S. Haseler, and A. Poglitsch, Phys. Rev. A 34, 493 (1986)].

${ }^{9}$ R. C. Gauthier, Appl. Phys. Lett. 67, 2269 (1995).

${ }^{10}$ Modern Developments in Fluid Dynamics, edited by S. Goldstein (Dover, New York, 1965), Vols. I and II. 